\title{
CONSTRAINTS ON VERY HIGH ENERGY EMISSION FROM GRB 130427A
}

\author{
E. Aliu ${ }^{1}$, T. Aune ${ }^{2}$, A. Barnacka ${ }^{3}$, M. Beilicke ${ }^{4}$, W. Benbow ${ }^{5}$, K. Berger $^{6}$, J. Biteau $^{7}$, J. H. Buckley $^{4}$, V. BugaeV $^{4}$,
} K. Byrum ${ }^{8}$, J. V Cardenzana ${ }^{9}$, M. Cerruti ${ }^{5}$, X. Chen $^{10,11}$, L. CiupiK ${ }^{12}$, V. Connaughton ${ }^{13}$, W. Cui ${ }^{14}$, H. J. Dickinson ${ }^{9}$,

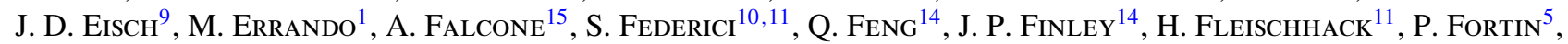
L. Fortson $^{16}$, A. Furniss ${ }^{7}$, N. Galante ${ }^{5}$, G. H. Gillanders ${ }^{17}$, S. Griffin ${ }^{18}$, S. T. Griffiths ${ }^{19}$, J. Grube ${ }^{12}$, G. Gyuk ${ }^{12}$,

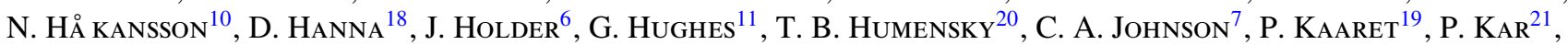

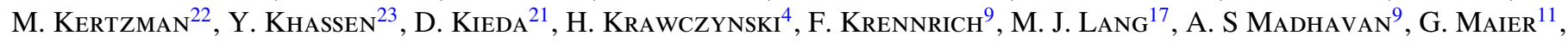
S. McArthur ${ }^{24}$, A. McCann ${ }^{25}$, K. Meagher ${ }^{26}$, J. Millis $^{27}$, P. Moriarty ${ }^{17,28}$, R. Mukherjee ${ }^{1}$, D. Nieto $^{20}$, A. O’Faoláin de Bhróithe ${ }^{11}$, R. A. Ong ${ }^{2}$, A. N. Otte ${ }^{26}$, N. Park ${ }^{24}$, M. Pohl $^{10,11}$, A. Popkow ${ }^{2}$, H. Prokoph $^{11}$, E. Pueschel ${ }^{23}$, J. QuinN ${ }^{23}$, K. Ragan ${ }^{18}$, J. Rajotte ${ }^{18}$, L. C. Reyes ${ }^{29}$, P. T. Reynolds ${ }^{30}$, G. T. Richards ${ }^{26}$, E. RoACHE ${ }^{5}$, G. H. SEMbroski ${ }^{14}$, K. Shahinyan ${ }^{16}$, A. W. Smith ${ }^{21}$, D. Staszak ${ }^{18}$, I. Telezhinsky ${ }^{10,11}$, J. V. Tucci ${ }^{14}$, J. Tyler ${ }^{18}$, A. VARlotTa $^{14}$, V. V. VAssilieV ${ }^{2}$, S. Vincent ${ }^{11}$, S. P. Wakely ${ }^{24}$, O. M. Weiner ${ }^{20}$, A. Weinstein ${ }^{9}$, R. Welsing ${ }^{11}$, A. Wilhelm $^{10,11}$, D. A. Williams ${ }^{7}$, B. Zitzer ${ }^{8}$, J. E. McEnery ${ }^{31,32}$, J. S. Perkins ${ }^{31}$, P. Veres ${ }^{33,34}$, and S. Zhu ${ }^{32}$

${ }^{1}$ Department of Physics and Astronomy, Barnard College, Columbia University, NY 10027, USA

${ }^{2}$ Department of Physics and Astronomy, University of California, Los Angeles, CA 90095, USA; aune@ astro.ucla.edu

${ }^{3}$ Harvard-Smithsonian Center for Astrophysics, 60 Garden Street, Cambridge, MA 02138, USA

${ }^{4}$ Department of Physics, Washington University, St. Louis, MO 63130, USA

${ }^{5}$ Fred Lawrence Whipple Observatory, Harvard-Smithsonian Center for Astrophysics, Amado, AZ 85645, USA

${ }^{6}$ Department of Physics and Astronomy and the Bartol Research Institute, University of Delaware, Newark, DE 19716, USA

${ }^{7}$ Santa Cruz Institute for Particle Physics and Department of Physics, University of California, Santa Cruz, CA 95064, USA

${ }^{8}$ Argonne National Laboratory, 9700 S. Cass Avenue, Argonne, IL 60439, USA

${ }^{9}$ Department of Physics and Astronomy, Iowa State University, Ames, IA 50011, USA

${ }^{10}$ Institute of Physics and Astronomy, University of Potsdam, 14476 Potsdam-Golm, Germany

${ }^{11}$ DESY, Platanenallee 6, 15738 Zeuthen, Germany

12 Astronomy Department, Adler Planetarium and Astronomy Museum, Chicago, IL 60605, USA

${ }^{13}$ Center for Space Plasma and Aeronomic Research (CSPAR), University of Alabama in Huntsville, Huntsville, AL 35899, USA

${ }_{14}$ Department of Physics and Astronomy, Purdue University, West Lafayette, IN 47907, USA

${ }^{15}$ Department of Astronomy and Astrophysics, 525 Davey Lab, Pennsylvania State University, University Park, PA 16802, USA

${ }_{16}$ School of Physics and Astronomy, University of Minnesota, Minneapolis, MN 55455, USA

${ }^{17}$ School of Physics, National University of Ireland Galway, University Road, Galway, Ireland

${ }^{18}$ Physics Department, McGill University, Montreal, QC H3A 2T8, Canada

${ }^{19}$ Department of Physics and Astronomy, University of Iowa, Van Allen Hall, Iowa City, IA 52242, USA

${ }^{20}$ Physics Department, Columbia University, New York, NY 10027, USA

${ }^{21}$ Department of Physics and Astronomy, University of Utah, Salt Lake City, UT 84112, USA

22 Department of Physics and Astronomy, DePauw University, Greencastle, IN 46135-0037, USA ${ }^{23}$ School of Physics, University College Dublin, Belfield, Dublin 4, Ireland

${ }^{24}$ Enrico Fermi Institute, University of Chicago, Chicago, IL 60637, USA

${ }^{25}$ Kavli Institute for Cosmological Physics, University of Chicago, Chicago, IL 60637, USA

${ }^{26}$ School of Physics and Center for Relativistic Astrophysics, Georgia Institute of Technology, 837 State Street NW, Atlanta, GA 30332-0430, USA

${ }^{27}$ Department of Physics, Anderson University, 1100 East 5th Street, Anderson, IN 46012, USA

${ }^{28}$ Department of Life and Physical Sciences, Galway-Mayo Institute of Technology, Dublin Road, Galway, Ireland

${ }^{29}$ Physics Department, California Polytechnic State University, San Luis Obispo, CA 94307, USA

${ }^{30}$ Department of Applied Physics and Instrumentation, Cork Institute of Technology, Bishopstown, Cork, Ireland

${ }^{31}$ NASA Goddard Space Flight Center, Greenbelt, MD 20771, USA

32 Department of Physics and Department of Astronomy, University of Maryland, College Park, MD 20742, USA; sjzhu@umd.edu

${ }^{33}$ Department of Physics, George Washington University, Washington, DC 20052, USA; veres@email.gwu.edu

${ }^{34}$ Department of Astronomy and Astrophysics, Department of Physics, and Center for Particle and Gravitational Astrophysics, Pennsylvania State University, University Park, PA 16802, USA

Received 2014 June 25; accepted 2014 September 24; published 2014 October 10

\begin{abstract}
Prompt emission from the very fluent and nearby $(z=0.34)$ gamma-ray burst GRB 130427A was detected by several orbiting telescopes and by ground-based, wide-field-of-view optical transient monitors. Apart from the intensity and proximity of this GRB, it is exceptional due to the extremely long-lived high-energy (100 MeV to $100 \mathrm{GeV}$ ) gamma-ray emission, which was detected by the Large Area Telescope on the Fermi Gamma-Ray Space Telescope for $\sim 70 \mathrm{ks}$ after the initial burst. The persistent, hard-spectrum, high-energy emission suggests that the highest-energy gamma rays may have been produced via synchrotron self-Compton processes though there is also evidence that the high-energy emission may instead be an extension of the synchrotron spectrum. VERITAS, a ground-based imaging atmospheric Cherenkov telescope array, began follow-up observations of GRB 130427A $\sim 71 \mathrm{ks}(\sim 20 \mathrm{hr})$ after the onset of the burst. The GRB was not detected with VERITAS; however, the high elevation of the observations, coupled with the low redshift of the GRB, make VERITAS a very sensitive probe of the emission from GRB 130427A for $E>100 \mathrm{GeV}$. The non-detection and consequent upper limit derived place constraints on the synchrotron self-Compton model of high-energy gamma-ray emission from this burst.
\end{abstract}

Key word: gamma-ray burst: individual (GRB 130427A)

Online-only material: color figures 


\section{INTRODUCTION}

Gamma-ray bursts (GRBs) are commonly thought to result from collapsing massive stars or merging compact objects which form a black hole or neutron star. In the standard GRB model (see, for example, Piran 1999), the initial bright prompt emission is produced within a relativistic jet after it escapes through the stellar envelope and could produce radiation via a number of processes including internal shocks, magnetic reconnection, or hydromagnetic turbulence. As the ejecta sweep up external material, forward and reverse shocks are created that can accelerate charged particles, producing $\mathrm{MeV}$ to $\mathrm{GeV}$ gammaray photons via synchrotron radiation. It has been suggested that GRBs might also create detectable fluxes of high-energy photons at later times via synchrotron self-Compton or external Compton processes (Zhang \& Mészáros 2001; Wang et al. 2001; Beloborodov 2005; Wang et al. 2006). In addition, the external shocks themselves could produce very high energy (VHE; $E>100 \mathrm{GeV}$ ) photons via the inverse Compton mechanism (Meszaros \& Rees 1994; Dermer et al. 2000; Fan et al. 2008; Sari \& Esin 2001). These non-thermal processes could produce photons with energies as high as $\sim 1 \mathrm{TeV}$ in the early afterglow phase of the GRB.

The extraordinary GRB 130427A was initially detected at 07:47:06.42 UTC (von Kienlin 2013) on 2013 April 27 by the Gamma-ray Burst Monitor (GBM; Meegan et al. 2009) on board the Fermi Gamma-Ray Space Telescope. This detection triggered an autonomous repoint request that kept the burst in the field of view (FoV) of the Large Area Telescope (LAT; Atwood et al. 2009) for $2.5 \mathrm{hr}$ except during periods of Earth occultation (Ackermann et al. 2014). The Burst Alert Telescope (BAT; Barthelmy et al. 2005) on board the Swift observatory independently triggered on this burst at 07:47:57 UTC (Maselli et al. 2014). The preliminary $15-350 \mathrm{keV}$ BAT light curve showed an extremely bright burst with a highly structured peak lasting $20 \mathrm{~s}$ and displaying a maximum count rate of approximately 100,000 counts per second (Maselli et al. 2013).

Levan et al. (2013) determined that the GRB was associated with a Type IC supernova (SN 2013cq) in a galaxy at a redshift of $z=0.34$. The average redshift of Swift-detected long GRBs is $z>2$ (Gehrels et al. 2009). GRB 130427A had the highest prompt fluence yet recorded by Konus-WIND (20-1200 keV; Golenetskii et al. 2013) and Fermi-GBM (von Kienlin 2013), as well as the longest-lasting emission and highest observed photon energy $(95 \mathrm{GeV})$ from a GRB yet recorded by the FermiLAT (Ackermann et al. 2014). HAWC, a wide FoV, high duty cycle, water Cherenkov detector currently under construction and sensitive to gamma rays in the $\mathrm{GeV}-\mathrm{TeV}$ energy range (Abeysekara et al. 2014), did not detect prompt VHE emission from GRB 130427A (Lennarz \& Taboada 2013).

It has long been predicted that GRBs could emit gamma rays at energies above $100 \mathrm{GeV}$. GRB 080916C (Atwood et al. 2013) and GRB 130427A both produced photons with energies above $100 \mathrm{GeV}$, but the detected photon energies were lower due to the cosmological redshift of the bursts. No direct detection of $>100 \mathrm{GeV}$ photons has yet been achieved even though significant effort has been put into searching for such emission (Connaughton et al. 1997; Aharonian et al. 2009; Albert et al. 2007; Acciari et al. 2011; Atkins et al. 2005).

A simple extrapolation of the late-time LAT light curve to very high energies, taking into account extragalactic background light (EBL; Gould \& Schréder 1967) attenuation, indicates that current-generation IACT arrays were sensitive enough to detect
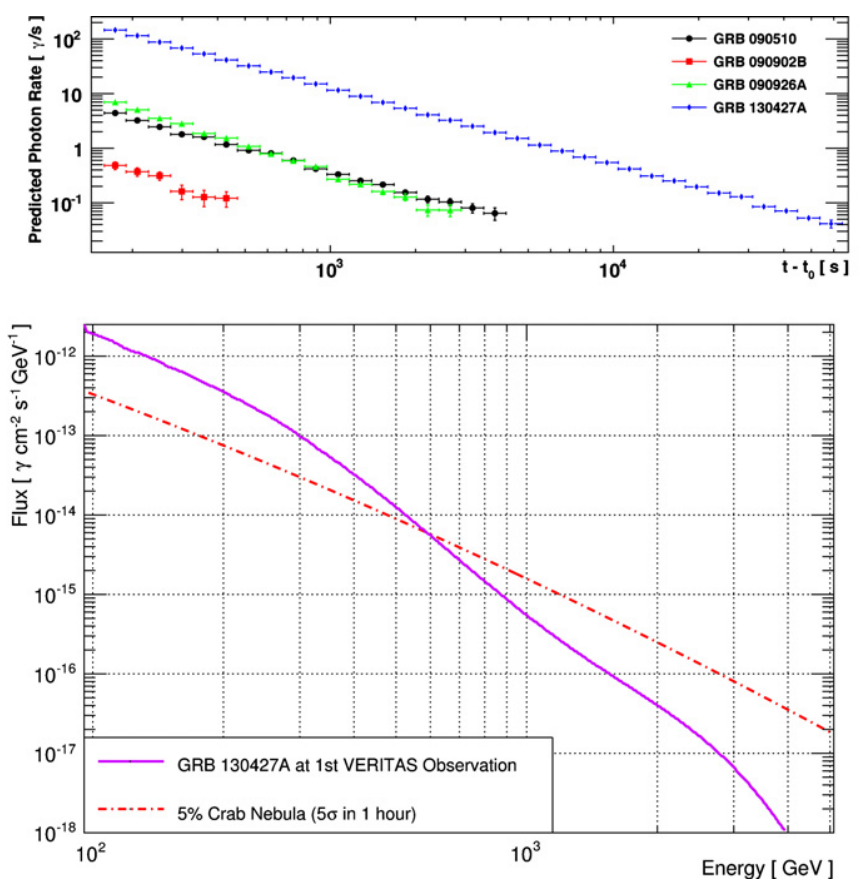

Figure 1. Upper panel shows predicted light curves for several fluent, LATdetected GRBs: GRB 090510 (De Pasquale et al. 2010), GRB 090902B (Abdo et al. 2009), GRB 090926A (Ackermann et al. 2011), and GRB 130427A (Ackermann et al. 2014), as they would be seen by VERITAS at energies greater than $100 \mathrm{GeV}$ assuming an elevation of $70 \mathrm{deg}$. Each bin in the upper panel is derived from a figure similar to that in the lower panel, which is a detail of the photon flux extrapolated from LAT data of GRB 130427A (including EBL absorption) over the period of the first VERITAS observation (see Table 1). The red dashed-dotted line is 5\% of the average Crab Nebula spectrum for reference. VERITAS is capable of detecting a 5\% Crab Nebula source over the duration of the first GRB 130427A observation $(\sim 1 \mathrm{hr})$. The lack of a detection by VERITAS suggests the presence of a spectral and/or temporal cutoff at high energies and late times, respectively.

(A color version of this figure is available in the online journal.)

GRB 130427A up to about a day after the onset of the burst. The top panel of Figure 1 shows the predicted light curves for several bright LAT-detected GRBs as they would appear to VERITAS, an IACT array sensitive to gamma rays above $100 \mathrm{GeV}$ and located in southern Arizona. All bins represent a detection of more than three standard deviations above background $(>3 \sigma)$. The predictions use the fluxes and spectra from the LAT measurements, specifically $d N / d t \propto t^{-1.35}$ and $d N / d E \propto E^{-2.2}$ (Ackermann et al. 2014) and include the absorption of gamma rays by the EBL according to the model of Gilmore et al. (2009). GRB 130427A, shown in blue, is by far the most promising candidate for a VHE detection by VERITAS. VERITAS made observations toward the direction of the GRB starting $\sim 20 \mathrm{hr}$ after the initial satellite detection but did not detect any emission from the burst. This Letter details those observations and places them in context with observations at other wavelengths, especially those made by the LAT. Additionally, constraints on the VHE emission obtained from this non-detection are discussed in the context of various emission models.

\section{OBSERVATIONS}

\subsection{VERITAS}

The energy range of the VERITAS array extends from $\sim 100 \mathrm{GeV}$ to several tens of TeV, overlapping with the energy range of the LAT (for an overview of VERITAS; see Holder et al. 2012). The VERITAS Collaboration has had a GRB observing 
Table 1

VERITAS Observations of GRB 130427A

\begin{tabular}{|c|c|c|c|c|c|c|c|c|}
\hline Date & $\begin{array}{c}t_{\text {start }} \\
(\mathrm{UTC})\end{array}$ & $\begin{array}{c}t_{\text {end }} \\
(\mathrm{UTC})\end{array}$ & $\begin{array}{c}\text { Exposure } \\
\text { (s) }\end{array}$ & $n_{\mathrm{on}}$ & $n_{\text {off }}$ & $\alpha^{\mathrm{a}}$ & $\begin{array}{c}\text { Significance }^{b} \\
(\sigma)\end{array}$ & Flux UL ${ }^{c}$ \\
\hline 2013 Apr 28 & $03: 32: 35$ & $04: 31: 16$ & 2925 & 165 & 1164 & 0.125 & 1.3 & $9.4 \times 10^{-12}$ \\
\hline 2013 Apr 29 & $03: 32: 59$ & $05: 33: 39$ & 5746 & 322 & 2120 & 0.143 & 1.1 & $6.6 \times 10^{-12}$ \\
\hline 2013 Apr 30 & $03: 22: 02$ & $06: 05: 40$ & 7814 & 402 & 2820 & 0.147 & -0.5 & $2.7 \times 10^{-12}$ \\
\hline Total & & & 16485 & 889 & 6104 & 0.141 & 0.9 & $3.3 \times 10^{-12}$ \\
\hline
\end{tabular}

Notes.

${ }^{a}$ Ratio of the signal region to background region.

b Significance calculated using Equation (17) of Li \& Ma (1983).

c $99 \%$ confidence-level upper limit on $\nu \mathrm{F}_{\nu}$ in erg $\mathrm{cm}^{-2} \mathrm{~s}^{-1}$.The upper limit is derived using the method of Rolke et al. (2005), quoted at 100 GeV, and calculated assuming an intrinsic GRB spectrum of $(\mathrm{d} N / \mathrm{d} E) \propto E^{-2.0}$ (as measured by the LAT) absorbed using the EBL model of Gilmore et al. (2009).

program since it began full array operations in 2007 and has performed more than 100 follow-up observations of GRBs detected by space-based instruments (Acciari et al. 2011). The VERITAS trigger system was upgraded in 2011 and the camera was upgraded one year later, resulting in improved sensitivity and a lower energy threshold (Kieda et al. 2013). It is estimated that VERITAS should be sensitive enough to detect bright and/or nearby GRBs.

At the time of the Fermi-GBM trigger (07:47 UTC), GRB 130427A was at a relatively favorable elevation of $52^{\circ}$ for VERITAS. Unfortunately, bright moonlight conditions (97\% full and $\sim 30^{\circ}$ above the horizon) precluded observations. Typical GRB follow-up observations are limited to three hours after a burst, but due to the extraordinary nature of GRB 130427A, VERITAS observations were initiated the following night, 2013 April 28, at 03:32:35 (UTC), $71.128 \mathrm{ks}$ after the Fermi-GBM trigger. Observations lasted for 59 minutes until moonrise. Observations continued on the following two nights, lasting $\sim 2$ and $\sim 2.5 \mathrm{hr}$, respectively (see Table 1 ). The average elevation of the GRB position at the time of the observations was $81^{\circ}$, resulting in a post-analysis energy threshold of $\sim 100 \mathrm{GeV}$.

\subsection{Fermi-LAT}

The LAT is a pair-conversion telescope that detects photons with energies from $20 \mathrm{MeV}$ to $>300 \mathrm{GeV}$ (Atwood et al. 2009). The GRB was within the LAT FoV (47.3 from the boresight) at the time of the trigger and remained in the FoV for the next $2.5 \mathrm{hr}$ due to the autonomous repoint request (except during times of Earth occultation). Once the observatory returned to survey mode, the GRB was in the FoV $\sim 40 \%$ of the time. During the first VERITAS observation $(71.0$ to $75.0 \mathrm{ks})$, the GRB was in the LAT FoV from 72.1 to $73.4 \mathrm{ks}$ and 73.5 to $74.9 \mathrm{ks}$; the last photon with energy greater than $1 \mathrm{GeV}$ was detected at $68.4 \mathrm{ks}$.

\section{ANALYSIS AND RESULTS}

\subsection{VERITAS}

The VERITAS data were analyzed with a standard VERITAS software package using event selection criteria optimized for a soft-spectrum $\left((d N / d E) \propto E^{-3.5}\right)$, weak (5\% Crab Nebula flux) point source, which roughly approximates the EBLabsorbed GRB spectrum. We decided, a priori, to analyze the data from each night's observations independently in addition to the complete data set together. We find no evidence for gamma-ray emission above $100 \mathrm{GeV}$ in any analysis. This result is confirmed by an analysis using an independent software package.
We derive upper limits on the VHE gamma-ray flux from GRB 130427A. The assumed spectral shape is extrapolated from the LAT observations, namely a power-law spectrum with a photon index of 2 with no intrinsic cutoff. The upper limits calculated for each time interval are given in Table 1.

\subsection{Fermi-LAT}

We analyzed the LAT data using an unbinned maximum likelihood method (as implemented in the Fermi Science Tools v9r30p1. ${ }^{35}$ ) The spectrum of the GRB is modeled as a power law and the background is modeled using the standard Galactic and isotropic diffuse models, specifically gal_2yearp7v6_v0.fits and iso_p7v6source.txt ${ }^{36}$; there were no LAT point sources in the region bright enough to warrant inclusion in the source model. Pass 7 Source class events within a $10^{\circ}$ region around the burst position (R.A. $=11^{\mathrm{h}} 32^{\mathrm{m}} 32.82^{\mathrm{s}}$ decl. $=+27^{\circ} 41^{\prime} 56^{\prime \prime}$.06, J2000; Perley 2013) were used with the standard zenith angle cut of $100^{\circ}$ (to limit contamination from the gamma-ray bright limb of the Earth) and the appropriate set of instrument response functions (P7_SOURCE_V6). The LAT emission decays smoothly after the first $\sim 20 \mathrm{~s}$; the energy flux light curve is well fitted with a single power law with a temporal index of $-1.17 \pm 0.06$, and the photon flux light curve is well fitted by a broken power law with a temporal index of $-0.85 \pm 0.08$ before $t-t_{0}=381 \pm 138$ $\mathrm{s}$ and a temporal index of $-1.35 \pm 0.08$ at later times (see Ackermann et al. 2014 for details of the analysis).

We considered the LAT emission between $10 \mathrm{ks}$ and $70 \mathrm{ks}$ after the burst. This was the last time interval before the VERITAS observations during which the LAT detected significant flux (Figure 2), as determined in (Ackermann et al. 2014). To test for spectral curvature, we also fitted the data with a power law with an exponential cutoff and with a broken power law. Neither of these models is statistically preferred over the simple power law. The spectrum of the GRB in this time interval is consistent with its spectrum earlier during the burst (the photon index is $2.2 \pm 0.2$; see Figure 2). The LAT data are best fitted with a power-law $(d N / d E)=N_{0}\left(E / E_{0}\right)^{-\gamma}$ where $E_{0}$, the decorrelation energy, is $826 \mathrm{MeV}, \gamma$ is $2.2 \pm 0.2$, and $N_{0}$ is $6.7 \pm 2.0 \times 10^{-11} \mathrm{~cm}^{-2} \mathrm{~s}^{-1} \mathrm{MeV}^{-1}$. This decorrelation energy is uniquely determined by the fit of the spectral index and integral flux over the energy range of the LAT and is the energy at which the normalization and spectral index are the least correlated.

\footnotetext{
35 http://fermi.gsfc.nasa.gov/ssc/data/analysis/software/

36 http://fermi.gsfc.nasa.gov/ssc/data/access/lat/BackgroundModels.html
} 

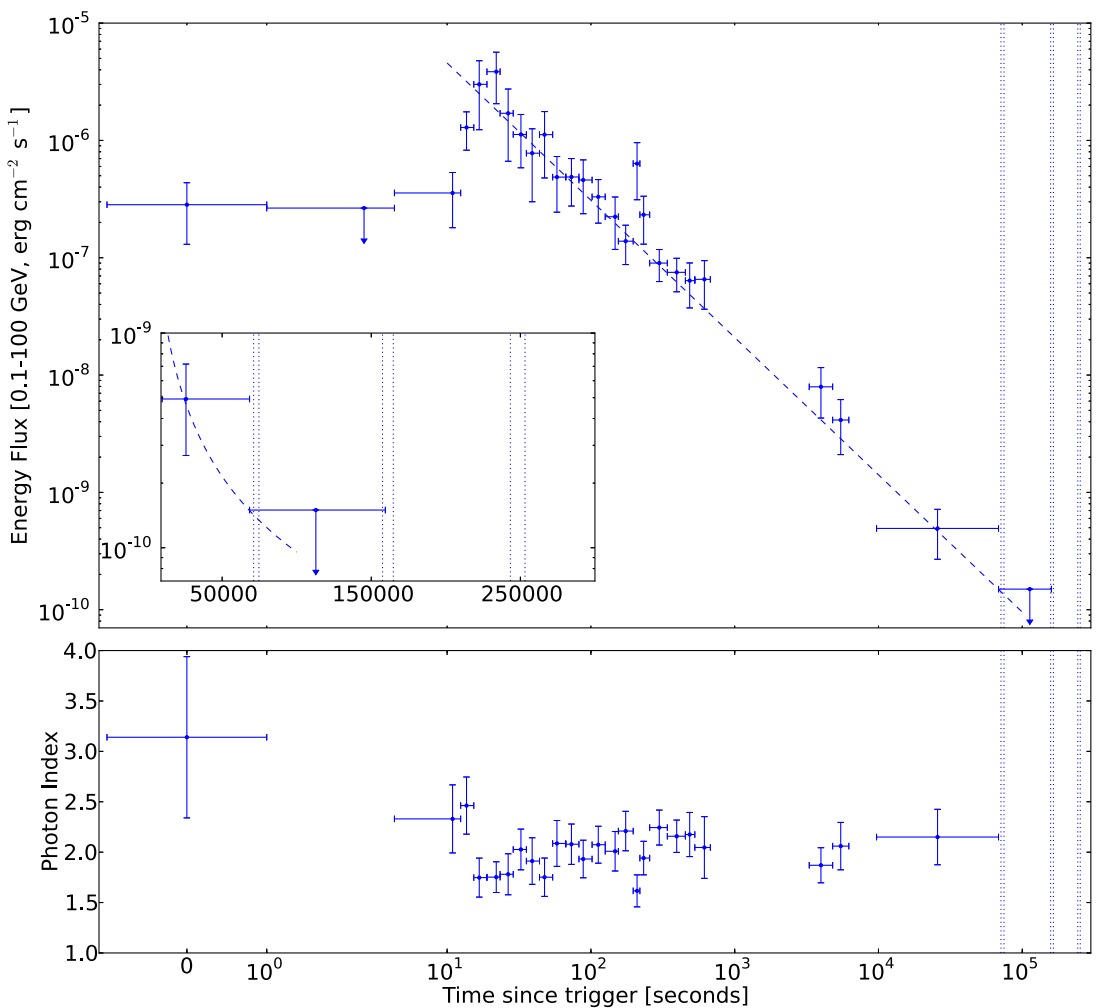

Figure 2. Upper panel shows the $0.1-100 \mathrm{GeV}$ light curve for GRB 130427A as measured by the LAT. The dashed line is a power-law fit to the light curve. The lower panel shows the LAT-measured photon index. These data have been shown previously in Ackermann et al. (2014). The vertical dotted lines indicate the times of the three VERITAS observations given in Table 1. The inset details these observations.

(A color version of this figure is available in the online journal.)

\section{DISCUSSION AND CONCLUSIONS}

The VERITAS upper limit and the last significant detection of high-energy emission by the LAT are not simultaneous. However, the late-time emission ( $>200 \mathrm{~s}$ ) measured by the LAT shows no deviation from a well-defined power-law behavior in both time and energy (see Figure 2), so we extrapolate the LAT data to the first VERITAS observing interval using the photon flux relation $d N / d t \propto t^{-1.35 \pm 0.08}$ measured by the LAT to create the joint VERITAS-LAT spectral energy distribution (SED) shown in Figure 3. While compatible with the extrapolation of the LAT measurement, the VERITAS upper limits disfavor a scenario in which there is an enhanced VHE component. Both synchrotron (e.g., Kouveliotou et al. 2013) and inverse Compton (e.g., Liu et al. 2013) scenarios have been proposed to explain the late-time, high-energy emission from GRB 130427A and we briefly examine these models in the context of the VERITAS upper limit.

Ackermann et al. (2014) noted that the synchrotron interpretation is problematic for this burst due to the observed latetime, high-energy photons, which contradict the robust limits obtained from a simple interpretation of the radiation produced in shocked plasma. However, Kouveliotou et al. (2013) find that both spectral and temporal extrapolations, from optical to multi$\mathrm{GeV}$ energies, are consistent with the synchrotron mechanism, though such an interpretation requires significant modifications to current models of particle acceleration in GRB afterglow shocks. In the context of the synchrotron model, we interpret the VERITAS upper limit in a scenario where the uniform magnetic field assumption in the shocked interstellar medium (ISM) is relaxed (Kumar et al. 2012), and the magnetic field decays

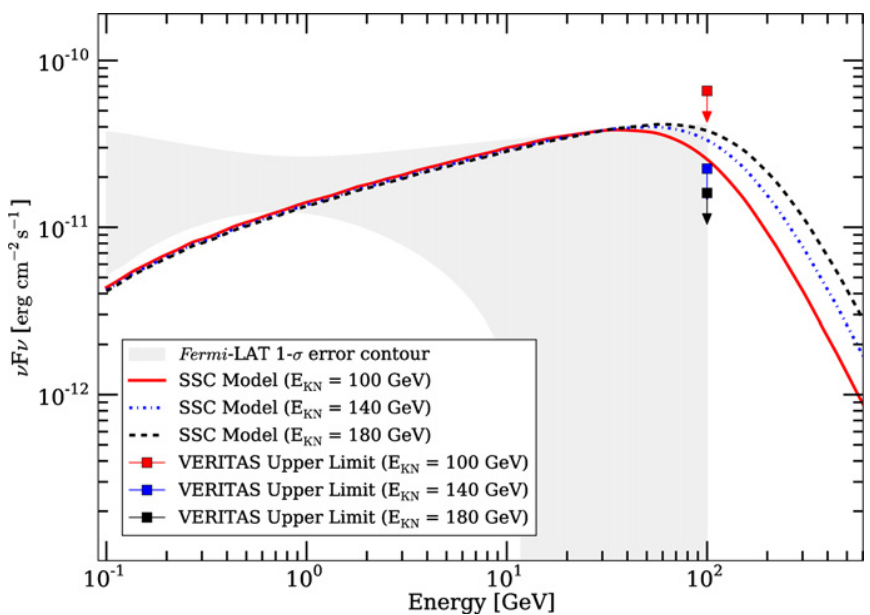

Figure 3. Joint VERITAS-LAT spectral energy distribution. The VERITAS upper limits are calculated assuming an SSC model (Sari \& Esin 2001) with an electron spectrum $(d N / d E) \propto E^{-2.45}$ and breaks at 100,140, and $180 \mathrm{GeV}$ (solid, dot-dashed, and dashed lines). The electron energy distribution is determined from the LAT-measured spectrum, as described in the text. This SED is then absorbed using the EBL model of Gilmore et al. (2009). The LAT data are best fitted with a power law with an index of $2.2 \pm 0.2$. The gray shaded region (the "bowtie") shows the one-sigma range of power-law models compatible with the LAT data after extrapolating from the last LAT time bin ( $10 \mathrm{ks}$ to $70 \mathrm{ks}$ ) into the VERITAS observing time ( $71 \mathrm{ks}$ to $75 \mathrm{ks}$ ) using the photon flux relation $(d N / d t) \propto t^{-1.35 \pm 0.08}$, which was obtained from fitting the late-time LAT data (Ackermann et al. 2014). The electron spectral index of the SSC models is determined from the error-weighted mean of the late-time spectral and temporal indices measured by the LAT.

(A color version of this figure is available in the online journal.) 
as a power law in the shocked region. Bearing in mind the assumptions of this model, the VERITAS non-detection can be associated with a cutoff in the synchrotron photon spectrum at $\sim 100 \mathrm{GeV}$. The theoretical limit on the synchrotron cutoff energy can be expressed as

$$
E_{\text {cut, syn }}=50 \mathrm{MeV}\left[\frac{\Gamma}{1+z}\right]\left(B_{w} / B_{0}\right)
$$

(Kumar et al. 2012). Here, $B_{w}$ is the magnetic field immediately behind the shock front and it carries a fraction $\left(\epsilon_{B}\right)$ of the shocked gas energy density. $B_{0}$ is the shock-compressed magnetic field of the ISM behind the thin shell associated with the shock itself $\left(B_{0} \approx 4 \Gamma B_{\mathrm{ISM}}\right)$. The Lorentz factor of the relativistic blast is derived from the self-similar phase of the Blandford-McKee model (Blandford \& McKee 1976) and can be written as $\Gamma \approx 10\left(E_{53} / n_{0}\right)^{1 / 8}\left(t / t_{V}\right)^{-3 / 8}$, where $E_{53}$ is the isotropic equivalent energy of the burst in units of $10^{53}$ ergs, $n_{0}=1 \mathrm{~cm}^{-3}$ is the density of the ISM, and $t_{V}$ is the time of the first VERITAS observation. We limit our discussion here to the ISM environment (Maselli et al. 2014), but we note that a wind (e.g. Perley et al. 2014) or hybrid environment ( $n \propto R^{-1.4 \pm 0.2}$, Kouveliotou et al. 2013) may instead reflect the conditions surrounding GRB 130427A. The VERITAS upper limit can thus constrain the $B_{w} / B_{0}$ ratio to be $\gtrsim 200$ where $B_{w}=\sqrt{32 \pi m_{p} \epsilon_{B} n_{0}} \Gamma c$ (Sari et al. 1998). This upper limit can in turn be used to constrain the magnetic field of the ISM in the GRB environment, which can be written as $B_{\text {ISM }} \lesssim 5 \mu \mathrm{G} E_{53}^{1 / 8} \epsilon_{B,-4}^{1 / 2} n_{0}^{3 / 8}\left(t / t_{V}\right)^{-3 / 8}$ where $\epsilon_{B,-4}=10^{-4} \epsilon_{B}$.

It is also possible that the late-time, high-energy emission detected by the LAT was produced from inverse-Compton scattering in a synchrotron self-Compton (SSC) scenario (Liu et al. 2013). The high-energy emission in an SSC model is synchrotron photons upscattered by the same electron population from which they were emitted (Sari \& Esin 2001). For reasonable parameters, it can be shown that the $0.1 \lesssim$ $E \lesssim 100 \mathrm{GeV}$ energy range falls between the characteristic $\left(E_{m}^{\mathrm{SSC}} \approx 2 \gamma_{m}^{2} E_{m} \approx 25 \mathrm{keV}\left(t / t_{V}\right)^{-9 / 4}\right)$ and cooling $\left(E_{c}^{\mathrm{SSC}} \approx\right.$ $\left.2 \gamma_{c}^{2} E_{c} \approx 400 \mathrm{GeV}\left(t / t_{V}\right)^{1 / 4}\right) \operatorname{SSC}$ energies $\left(\gamma_{m}\right.$ and $\gamma_{c}$ are the electron Lorentz factors at the minimum injection energy and the cooling energy, respectively). At late times, the LAT measures a photon index of $2.2 \pm 0.2$ and a temporal index of $-1.35 \pm 0.08$. Under the SSC model, both quantities can be used to obtain the momentum power-law index for the shockaccelerated electrons. For the input to the model, we use the error-weighted mean of the electron power-law indices determined by each method to obtain a shocked electron power-law index of $p=2.45$. It should be noted that though this choice of electron index is appropriate given the data, the spectral and temporal flux indices obtained from the SSC model with this assumption are only consistent with the LAT measurements at the level of two standard deviations. The Klein-Nishina energy is $E_{\mathrm{KN}}=\Gamma \gamma_{c} m_{e} c^{2} /(1+z) \approx 180 \mathrm{GeV}\left(t / t_{V}\right)^{1 / 4}$ (Guetta \& Granot 2003), where $\Gamma \approx 10\left(t / t_{V}\right)^{-3 / 8}$ is the bulk Lorentz factor of the forward shock. Above this energy, the electron-photon scattering cross section is reduced, resulting in a softening of the spectrum.

Figure 3 shows the expected flux from SSC models fitted to the late-time $\left(t-t_{0}>10 \mathrm{ks}\right)$ LAT-detected emission and with breaks at 100,140 , and $180 \mathrm{GeV}$. The SSC models used here are taken from the slow-cooling scenario described in Sari \& Esin (2001). Also plotted is the one sigma range of power-law models compatible with the LAT data from the last LAT time bin $\left(10 \mathrm{ks}<t-t_{0}<70 \mathrm{ks}\right)$ extrapolated to the VERITAS observation time, as well as the VERITAS upper limits for the three spectral assumptions. The VERITAS upper limits are incompatible with a spectral break above $\sim 120 \mathrm{GeV}$, or the absence of a break entirely. When the SSC model spectrum, which is determined from the temporally extrapolated LAT data, is extrapolated to energies above $\sim 100 \mathrm{GeV}$ in the model, the predicted flux conflicts with the upper limits obtained with VERITAS. This indicates that the simple single zone SSC model is not an accurate description of GRB 130427A at energies greater than $\sim 100 \mathrm{GeV}$. It should be noted that another possible explanation for the break is a pair production cutoff of $100 \mathrm{GeV}$ photons with $\sim 100 \mathrm{keV}$ photons; however, we find the optical depth for this process is very low $\tau_{\gamma \gamma} \sim 10^{-5}$. Thus we conclude that the most plausible interpretation in the framework of an inverse-Compton scenario is that we are observing the Klein-Nishina cutoff below the VHE range. Results presented in this work, combined with observations of GRB 130427A at lower energies, suggest a single dominant component in the afterglow. In order for this SSC interpretation to work, we need a fine tuning of the underlying physical parameters to: (1) have an SSC-dominated afterglow from the earliest times, or (2) transition smoothly from synchrotron to SSC-dominated regimes at late times. For this reason we prefer the synchrotron interpretation of GRB 130427A.

The VERITAS observations of GRB 130427A, even at $\sim 20 \mathrm{hr}$ after the burst, meaningfully constrain synchrotron and inverse Compton emission models that seek to explain the late-time, high-energy emission observed by the LAT. Although it is estimated that a burst as nearby as GRB 130427A will occur only once every several decades, it has been shown that bright bursts even out to $z \approx 2$ could be detectable by VERITAS (Acciari et al. 2011). VERITAS continues to perform follow-up observations of satellite-detected GRBs and efforts to improve these observations are currently underway (Williams et al. 2011).

VERITAS is supported by grants from the U.S. Department of Energy Office of Science, the U.S. National Science Foundation, and the Smithsonian Institution, by NSERC in Canada, by Science Foundation Ireland (SFI 10/RFP/AST2748) and by STFC in the U.K. Additional support for observations of GRBs comes from NASA grant NNX12AE30G. We acknowledge the excellent work of the technical support staff at the Fred Lawrence Whipple Observatory and at the collaborating institutions in the construction and operation of the instrument.

The Fermi LAT Collaboration acknowledges generous ongoing support from a number of agencies and institutes that have supported both the development and the operation of the LAT as well as scientific data analysis. These include the National Aeronautics and Space Administration and the Department of Energy in the United States, the Commissariat à l'energie atomique and the Centre national de la recherche scientifique/Institut national de physique nucléaire et de physique des particules in France, the Agenzia Spaziale Italiana and the Istituto Nazionale di Fisica Nucleare in Italy, the Ministry of Education, Culture, Sports, Science, and Technology (MEXT), High Energy Accelerator Research Organization (KEK), and Japan Aerospace Exploration Agency (JAXA) in Japan, and the K. A. Wallenberg Foundation, the Swedish Research Council, and the Swedish National Space Board in Sweden.

Additional support for science analysis during the operations phase is gratefully acknowledged from the Istituto Nazionale di 
Astrofisica in Italy and the Centre National d'Études Spatiales in France.

\section{REFERENCES}

Abdo, A. A., Ackermann, M., Ajello, M., et al. 2009, ApJL, 706, L138 Abeysekara, A. U., Alfaro, R., Alvarez, C., et al. 2014, in Proc. of 33rd ICRC, Rio de Janeiro, Brazil, ed. A. Saa (arXiv:1410.1536)

Acciari, V. A., Aliu, E., Arlen, T., et al. 2011, ApJ, 743, 62

Ackermann, M., Ajello, M., Asano, K., et al. 2011, ApJ, 729, 114

Ackermann, M., Ajello, M., Asano, K., et al. 2014, Sci, 343, 42

Aharonian, F., Akhperjanian, A. G., Barres de Almeida, U., et al. 2009, A\&A, 495,505

Albert, J., Aliu, E., Anderhub, H., et al. 2007, ApJ, 667, 358

Atkins, R., Benbow, W., Berley, D., et al. 2005, ApJ, 630, 996

Atwood, W. B., Abdo, A. A., Ackermann, M., et al. 2009, ApJ, 697, 1071

Atwood, W. B., Baldini, L., Bregeon, J., et al. 2013, ApJ, 774, 76

Barthelmy, S. D., Barbier, L. M., Cummings, J. R., et al. 2005, SSRv, 120, 143

Beloborodov, A. M. 2005, ApJL, 618, L13

Blandford, R. D., \& McKee, C. F. 1976, PhFl, 19, 1130

Connaughton, V., Ackerlof, C. W., Barthelmy, S., et al. 1997, ApJ, 479, 859

De Pasquale, M., Schady, P., Kuin, N. P. M., et al. 2010, ApJL, 709, L146

Dermer, C. D., Chiang, J., \& Mitman, K. E. 2000, ApJ, 537, 785

Fan, Y. Z., Piran, T., Narayan, R., \& Wei, D. M. 2008, MNRAS, 384, 1483

Gehrels, N., Ramirez-Ruiz, E., \& Fox, D. B. 2009, ARA\&A, 47, 567

Gilmore, R. C., Madau, P., Primack, J. R., Somerville, R. S., \& Haardt, F 2009, MNRAS, 399, 1694

Golenetskii, S., Aptekar, R., Frederiks, D., et al. 2013, GCN, 1448
Gould, R., \& Schréder, G. 1967, PhR, 155, 1404

Guetta, D., \& Granot, J. 2003, MNRAS, 340, 115

Holder, J., Aliu, E., Arlen, T., et al. 2012, in Proc. 32nd, ICRC, 12, 137 (arXiv:1111.1225)

Kieda, D. B., Acciari, V. A., Aliu, E., et al. 2013, in Proc. of 33rd ICRC, Rio de Janeiro, Brazil, ed. A. Saa (arXiv:1308.4849)

Kouveliotou, C., Granot, J., Racusin, J. L., et al. 2013, ApJL, 779, L1

Kumar, P., Hernández, R. A., Bošnjak, Z., \& Duran, R. B. 2012, MNRAS, 427, L40

Lennarz, D., \& Taboada, I. 2013, GCN, 1454

Levan, A. J., Fruchter, A. S., Graham, J., et al. 2013, GCN, 14686

Li, T., \& Ma, Y. 1983, ApJ, 272, 317

Liu, R. Y., Wang, X. Y., \& Wu, X. F. 2013, ApJL, 773, L20

Maselli, A., Beardmore, A. P., Lien, A. Y., et al. 2013, GCN, 14448

Maselli, A., Melandri, A., Nava, L., et al. 2014, Sci, 343, 48

Meegan, C., Lichti, G., Bhat, P. N., et al. 2009, ApJ, 702, 791

Meszaros, P., \& Rees, M. J. 1994, MNRAS, 269, L41

Perley, D. A. 2013, GCN, 14494

Perley, D. A., Cenko, S. B., Corsi, A., et al. 2014, ApJ, 781, 37

Piran, T. 1999, PhR, 314, 575

Rolke, W. A., López, A. M., \& Conrad, J. 2005, NIMPA, 551, 493

Sari, R., \& Esin, A. A. 2001, ApJ, 548, 787

Sari, R., Piran, T., \& Narayan, R. 1998, ApJL, 497, L17

von Kienlin, A. 2013, GCN, 14473

Wang, X. Y., Dai, Z. G., \& Lu, T. 2001, ApJ, 556, 1010

Wang, X. Y., Li, Z., \& Mészáros, P. 2006, ApJL, 641, L89

Williams, D. A., Acciari, V. A., Aliu, E., et al. 2011, in Proc. of Gamma Ray Bursts 2010, ed. J. E. McEnery, J. L. Racusin, \& N. Gehrels (Annapolis, MD: AIP), 405

Zhang, B., \& Mészáros, P. 2001, ApJ, 559, 110 